\title{
SIMILARITIES/DISSIMILARITIES ANALYSIS OF PROTEIN SEQUENCES BASED ON PCA-FFT
}

\author{
PENGYAO PING $,{ }^{*}, \dagger$ XIANYOU ZHU ${ }^{\ddagger}$ and LEI WANG ${ }^{*}, \dagger, \S$ \\ ${ }^{*}$ College of Information Engineering, Xiangtan University \\ Yuhu District, Xiangtan, Hunan 411105, P. R. China \\ ${ }^{\dagger}$ Key Laboratory of Intelligent Computing and Information Processing \\ $\ddagger$ Department of Computer Science \\ Hengyang Normal University, 421008, P. R. China \\ \$364715358@qq.com
}

Received 5 January 2016

Revised 10 November 2016

Accepted 22 November 2016

Published 25 January 2017

\begin{abstract}
In this paper, a novel method to analyze the similarity/dissimilarity of protein sequences based on Principal Component Analysis-Fast Fourier Transformation (PCA-FFT) is proposed, in which, the PCA is utilized to transform protein sequences into time series and the FFT is utilized to analyze the time series while considering them as signals. To test the effectiveness of our newly proposed method, it is applied to analyze the similarity/dissimilarity of 16 different ND5 protein sequences and 29 different spike protein sequences, respectively. Furthermore, the correlation analysis is presented for comparing with others methods, and the simulation results show that it has better performances in the aspects of computation complexity and recognition degree than some existing methods.
\end{abstract}

Keywords: Time Series; Similarity/Dissimilarity Analysis; Principal Component Analysis; Fast Fourier Transformation; Protein Sequence.

\section{Introduction}

Homology is the relationship between biological structures or sequences that derived from a common ancestor. Homology among proteins or DNAs is typically inferred from their sequences similarity. Doing homology analysis is helpful for scientists' study on the function of DNA, proteins and cells. Alignments of sequences are used to discover the homologous regions. There are a variety of reasons why protein alignment is almost always preferable to DNA alignment. ${ }^{1}$ Firstly, redundant codons mean $\sim 1 / 3$ of DNA mutations often do not matter. Secondly, protein sequences are easier to achieve statistically significant alignment by comparing with DNA sequences, which are made of a larger alphabet of characters of four nucleotides

\section{$\S$ Corresponding author.}

This is an Open Access article published by World Scientific Publishing Company. It is distributed under the terms of the Creative Commons Attribution 4.0 (CC-BY) License. Further distribution of this work is permitted, provided the original work is properly cited. 
because you are much less likely to get a match by chance. Thirdly, the DNA database is cluttered with non-coding sequences, and not all amino acid mutations are equally harmful to protein structure. Homology analysis is one of the hot topics in the area of protein sequences analysis. Up till now, lots of methods have been proposed for the homology analysis of protein sequences. ${ }^{2,3}$ The graphical technique which was first proposed by Hamori ${ }^{4}$ over 30 years ago was mainly used for the graphical representation of DNA. Currently, many researchers have proposed different methods for the graphical representation of protein sequences, which is a very powerful tool for visual comparison of protein sequences. For example, Hou et al. ${ }^{5}$ outlined a 3D graphical representation based on the classification of amino acids by different physicochemical properties, and then computed the similarities among different protein sequences. Elmaatv et al. ${ }^{6}$ proposed a 3D graphical representation of protein sequences based on three physicochemical properties of amino acid side chains and calculated similarities among nine ND5 protein sequences. Wen et al. ${ }^{7}$ raised a $2 \mathrm{D}$ graphical representation of protein sequence according to the physicochemical properties of amino acids and considered the numerical characterization of mutations and analyzed the similarities of nine ND5 proteins. Zhang et al. ${ }^{8}$ used the occurrence frequency of 20 amino acids and took the new numerical characteristic of 2D graphical representation to compute the similarities of ND5 proteins of nine species based on the Euclidean distance. Wu et al. ${ }^{9}$ proposed a two-dimensional graph, called "2D-MH" to compare the evolutionary distances between any number of protein sequences. Yao et al. ${ }^{10}$ introduced a dynamic $2 \mathrm{D}$ graphical representation based on physicochemical properties of amino acids and compared the sequences of nine ND5 proteins. $\mathrm{Mu}$ et $a .^{11}{ }^{11}$ compared the similarity/dissimilarity of protein sequences according to the distance frequency of amino acids. Yao et al. ${ }^{12}$ outlined a 2D graphical representation of protein sequences based on two classifications of amino acids. Liu et al. ${ }^{13}$ proposed an alignment-free method based on the relative Lempel-Ziv complexity to compare biological sequences. Liu et al. ${ }^{14}$ constructed a PH Curve based on two kinds of physicochemical properties of amino acids to analyze the similarity/dissimilarity of protein sequences. Lakkani et al. ${ }^{15}$ proposed a numerical method to measure the degree of similarity sequences of protein sequences based on $2 \mathrm{D}$ and $3 \mathrm{D}$ adjacency matrices. Ma et al. ${ }^{16}$ introduced a graphical representation of protein based on a novel iterated function system. Yao et al. ${ }^{17}$ proposed a $3 \mathrm{D}$ graphical representation of protein sequences based on three kinds of physical-chemical properties of amino acids.

For those existing graphical representations of protein sequences given above, the main drawbacks are that, the higher the dimension of the protein sequence graphs, the heavier the computation complexity, or the lower the recognition degree of the protein sequence graphs. Principal Component Analysis (PCA) is a standard tool in multivariate data analysis to reduce the number of dimensions, which has been proven effective in the process of protein sequence analysis. ${ }^{18-20}$ Therefore, in order to overcome the main drawbacks of existing graphical representation methods given above, in this paper, we proposed a novel graphical representation of protein 
sequences, in which, the PCA is utilized to transform protein sequences into time series and the Fast Fourier Transformation (FFT), ${ }^{21,22}$ a common used signal analysis tool, is utilized to analyze the time series while considering them as signals. Additionally, a novel method based on PCA-FFT is designed to analyze the similarity/dissimilarity of protein sequences, which has lower computation complexity and higher recognition degree of protein sequence graphs than the existing methods. To validate the effectiveness of our method, we utilize it to analyze the similarity/dissimilarity of the 16 ND5 proteins spike proteins respectively. Moreover, comparing with existing methods, our new method is better at the same time, since it doesn't need to construct any complex matrixes when it is utilized to analyze the similarity/dissimilarity of protein sequences.

\section{Material and Methods}

\subsection{Amino acids and their properties}

Proteins are composed of 20 different amino acids. In order to analyze the similarity/dissimilarity of the protein sequences, the properties of the amino acids are considered first. Amino acids have many different physicochemical properties and biological characteristics including the molecular weight $(\mathrm{mW})$, Hydropathy index $(h I)$, solubility $(\mathrm{S})$, van der Waals radius of side chains (vR), the $p K a$ value for terminal amino acid groups $\mathrm{COOH}(P k 1)$, the $p K a$ value for terminal amino acid groups $\mathrm{NH}_{3}^{+}(p K 2)$, isoelectric point $(p I)$, the number of triplet codons $(c N)$, frequency of human proteins $(F)$ and so on. The nine properties of these amino acids are collected and illustrated in Table $1 .^{23-25}$

Table 1. The full list of 20 amino acids and their different properties.

\begin{tabular}{lcrrccccccr}
\hline Amino acid & Symbol & $\mathrm{mW}$ & \multicolumn{1}{c}{$h I$} & $p k 1$ & $p K 2$ & $p I$ & $\mathrm{~S}$ & $c N$ & $F(\%)$ & $\mathrm{vR}$ \\
\hline Alanine & $\mathrm{A}$ & 89.079 & 1.8 & 2.34 & 9.69 & 6.01 & 167.2 & 4 & 7.8 & 67 \\
Cysteine & $\mathrm{C}$ & 121.145 & 2.5 & 1.96 & 10.28 & 5.07 & 0 & 2 & 1.9 & 86 \\
Aspartic acid & $\mathrm{D}$ & 133.089 & -3.5 & 1.88 & 9.6 & 2.77 & 5 & 2 & 5.3 & 91 \\
Glutamic acid & $\mathrm{E}$ & 147.116 & -3.5 & 2.19 & 9.67 & 3.22 & 8.5 & 2 & 6.3 & 109 \\
Phenylalanine & $\mathrm{F}$ & 165.177 & 2.8 & 1.83 & 9.13 & 5.48 & 27.6 & 2 & 3.9 & 135 \\
Glycine & $\mathrm{G}$ & 75.052 & -0.4 & 2.34 & 9.6 & 5.97 & 249.9 & 4 & 7.2 & 48 \\
Histidine & $\mathrm{H}$ & 155.141 & -3.2 & 1.82 & 9.17 & 7.59 & 0 & 2 & 2.3 & 118 \\
Isoleucine & $\mathrm{I}$ & 131.16 & 4.5 & 2.36 & 9.68 & 6.02 & 34.5 & 3 & 5.3 & 124 \\
Lysine & $\mathrm{K}$ & 146.17 & -3.9 & 2.18 & 8.95 & 9.74 & 739 & 2 & 5.9 & 135 \\
Leucine & $\mathrm{L}$ & 131.16 & 3.8 & 2.36 & 9.6 & 5.98 & 21.7 & 6 & 9.1 & 124 \\
Methionine & $\mathrm{M}$ & 149.199 & 1.9 & 2.28 & 9.21 & 5.74 & 56.2 & 1 & 2.3 & 124 \\
Aspargine & $\mathrm{N}$ & 132.104 & -3.5 & 2.02 & 8.8 & 5.41 & 28.5 & 2 & 4.3 & 96 \\
Proline & $\mathrm{P}$ & 115.117 & 1.6 & 1.99 & 10.96 & 6.48 & 1620 & 4 & 5.2 & 90 \\
Glutamine & $\mathrm{Q}$ & 146.131 & -3.5 & 2.17 & 9.13 & 5.65 & 7.2 & 2 & 4.2 & 114 \\
Arginine & $\mathrm{R}$ & 174.188 & -4.5 & 2.17 & 9.04 & 10.76 & 855.6 & 6 & 5.1 & 148 \\
Serine & $\mathrm{S}$ & 105.078 & -0.8 & 2.21 & 9.15 & 5.68 & 422 & 6 & 6.8 & 73 \\
Tyrosine & $\mathrm{T}$ & 119.105 & -0.7 & 2.11 & 9.62 & 5.87 & 13.2 & 4 & 5.9 & 93 \\
Valine & $\mathrm{V}$ & 117.133 & 4.2 & 2.32 & 9.62 & 5.97 & 58.1 & 4 & 6.6 & 105 \\
Tryptophan & $\mathrm{W}$ & 204.213 & -0.9 & 2.38 & 9.39 & 5.89 & 13.6 & 1 & 1.4 & 163 \\
Threonine & $\mathrm{Y}$ & 181.176 & -1.3 & 2.2 & 9.11 & 5.66 & 0.4 & 2 & 3.2 & 141 \\
\hline
\end{tabular}




\subsection{Principal component analysis}

PCA is a common technique for dimensionality reduction and finding patterns in high dimension data. Proposed by Pearson in 1901, it is based on computation of low-dimensional representation of a high-dimensional dataset that maximizes the total scatter, which is optimal in reconstruction. ${ }^{26}$ The following describes the description in steps:

Step 1. The $m$ samples can be represented by a set $\Psi=\left\{X_{1}, X_{2}, \ldots, X_{m}\right.$. For each sample, which has $n$ indicators, it can be described as $X_{i}=\left\{x_{i 1} x_{i 2} \ldots, x_{i n}\right\}$ for $i \epsilon\{1,2, \ldots, m\}$, and then a $m \times n$ matrix $X$ can be constructed with all the observations as follows:

$$
X=\left[\begin{array}{c}
X_{1} \\
X_{2} \\
\vdots \\
X_{m}
\end{array}\right]=\left[\begin{array}{cccc}
x_{11} & x_{12} & \ldots & x_{1 n} \\
x_{21} & x_{22} & \ldots & x_{2 n} \\
\vdots & \vdots & \vdots & \vdots \\
x_{m 1} & x_{m 2} & \ldots & x_{m n}
\end{array}\right] .
$$

Then, based on the matrix $X$, the standardized matrix $X^{*}$ can be obtained by the following procedure.

$$
X^{*}=\left[\begin{array}{c}
X_{1}^{*} \\
X_{2}^{*} \\
\vdots \\
X_{m}^{*}
\end{array}\right]=\left[\begin{array}{cccc}
x_{11}^{*} & x_{12}^{*} & \ldots & x_{1 n}^{*} \\
x_{21}^{*} & x_{22}^{*} & \ldots & x_{2 n}^{*} \\
\vdots & \vdots & \vdots & \vdots \\
x_{m 1}^{*} & x_{m 2}^{*} & \ldots & x_{m n}
\end{array}\right],
$$

where $X_{i}^{*}=\left(x_{i 1}^{*}, x_{i 2}^{*}, \ldots, x_{i n}^{*}\right), x_{i j}^{*}=\left(x_{i j}-\bar{x}_{j}\right) / \sqrt{\operatorname{var}\left(x_{j}\right)}$ is the value of the element in the $X^{*}$ after standardizing, $\bar{x}_{j}=(1 / n) \sum_{i=1}^{n} x_{i j}$ and $\operatorname{var}\left(x_{j}\right)=(1 /(n-1))$ $\sum_{i=1}^{n}\left(x_{i j}-\bar{x}_{j}\right)^{2}$ is the average value and the variance of the $j$ th column of matrix $X$ respectively, for $i \in\{1,2, \ldots, m\}$ and $j \in\{1,2, \ldots, n\}$.

Step 2. Then we can obtain the correlation matrix $R$ based on the matrix $X^{*}$

$$
R=\left[\begin{array}{cccc}
r_{11} & r_{12} & \ldots & r_{1 n} \\
r_{21} & r_{22} & \ldots & r_{2 n} \\
\vdots & \vdots & \vdots & \vdots \\
r_{n 1} & r_{n 2} & \ldots & r_{n n}
\end{array}\right]
$$

where the value of element $r_{i j}$ in the matrix $R$ can be calculated as follows:

$$
r_{i j}=\frac{\sum_{k=1}^{m}\left(x_{k i}^{*}-\overline{x_{i}^{*}}\right)\left(x_{k j}^{*}-\overline{x_{j}^{*}}\right)}{\sqrt{\sum_{k=1}^{m}\left(x_{k i}^{*}-\overline{x_{i}^{*}}\right)^{2} \sum_{k=1}^{m}\left(x_{k j}^{*}-\bar{x}_{j}^{*}\right)^{2}}},
$$

where $\bar{x}_{i}^{*}=(1 / n) \sum_{j=1}^{n} x_{i j}^{*}$, for $i \in\{1,2, \ldots, n\}$ and $j \in\{1,2, \ldots, n\}$. 
Step 3. The $n$ eigenvalues $\lambda_{1} \geq \lambda_{2} \geq \cdots \geq \lambda_{n}>0$ of matrix $R$ and the corresponding $n$ eigenvectors $\mathbf{a}_{\mathbf{1}}, \mathbf{a}_{\mathbf{2}}, \ldots, \mathbf{a}_{\mathbf{n}}$ can be obtained,

$$
\mathbf{a}_{\mathbf{1}}=\left[\begin{array}{c}
a_{11} \\
a_{21} \\
\vdots \\
a_{n 1}
\end{array}\right], \quad \mathbf{a}_{2}=\left[\begin{array}{c}
a_{12} \\
a_{22} \\
\vdots \\
a_{n 2}
\end{array}\right], \ldots, \mathbf{a}_{\mathbf{n}}=\left[\begin{array}{c}
a_{1 n} \\
a_{21} \\
\vdots \\
a_{n n}
\end{array}\right] .
$$

Then we can get $\mathrm{n}$ principal components as follows:

$$
F_{i}=\mathbf{a}_{1 i} \times X_{1}^{*}+\mathbf{a}_{2 i} \times X_{2}^{*}+\cdots+\mathbf{a}_{n i} \times X_{m}^{*}, \quad(i=1,2, \ldots, n)
$$

Step 4. Compute the contribution rate $\mathrm{CR}_{i}$ and the accumulative contribution rate $\mathrm{ACR}_{i}$ of each principal component $F_{i}(i=1,2, \ldots, n)$, respectively:

$$
\begin{aligned}
\mathrm{CR}_{i} & =\frac{\lambda_{i}}{\sum_{k=1}^{n} \lambda_{k}}, \\
\mathrm{ACR}_{i} & =\sum_{k=1}^{i} C R_{k}
\end{aligned}
$$

Usually, we just retain the top $t$ principal components $F_{1} F_{2}, \ldots, F_{t}$, which correspond to the eigenvalues $\lambda_{1}, \lambda_{2}, \ldots, \lambda_{t}$, and the corresponding accumulated contribution rate should satisfy that $\mathrm{ACR}_{-} t \geq 85 \%$.

Step 5. Through plugging the elements in the matrix $X^{*}$ into the expression of $t$ principal components, we can obtain the score matrix $F$.

$$
F=\left[\begin{array}{cccc}
F_{11} & F_{12} & \ldots & F_{1 t} \\
F_{21} & F_{21} & \ldots & F_{2 t} \\
\vdots & \vdots & \vdots & \vdots \\
F_{m 1} & F_{m 2} & \ldots & F_{m t}
\end{array}\right]
$$

where $F_{i j}=\mathbf{a}_{1 i} x_{j 1}^{*}+\mathbf{a}_{2 i} x_{j 2}^{*}+\cdots+\mathbf{a}_{n i} x_{j n}^{*}$ for $i \in\{1,2, \ldots, m\}$ and $j \in\{1,2, \ldots, t\}$.

Therefore, we transform the original matrix $X$ into matrix $F$ that has reduced dimensionality, while not losing much information of the original data.

Step 6. The score matrix $F=\left[F_{i j}\right]_{m \times t}$ is a $m \times t$ matrix, we can obtain the $i$ th sample's total points $(\mathrm{TP}), \mathrm{TP}_{i}=\sum_{j=1}^{t} F_{i j} \times \mathrm{CR}_{j}$, where $\mathrm{CR}_{j}$ is the $j$ th eigenvalue's contribution rate.

\subsection{PCA of the amino acids}

As mentioned above, proteins are composed of 20 different amino acids, and each of the amino acid has many different properties. If we use all of the properties to 
analyze the protein sequences, we will have to handle the high-dimension matrices, and the computational effort will also be very large. PCA is an applicable algorithm for us to reduce the dimension of the amino acids' properties matrices. Here, we will apply the PCA algorithm to the nine properties of the 20 amino acids in Table 1 .

According to the procedures in Sec. 2.2, the 20 amino acids are the 20 samples, we can get their nine properties as the observations, and then, we obtain the matrix $X$. We can use the functions in the software Matlab to help us for PCA. We list the eigenvalues and the contribution rate $\mathrm{CR}$ and the accumulative contribution rate ACR in Table 2.

Table 2 shows that the accumulative contribution rate of first four eigenvalues is $0.8588>0.85$, so we can just retain the first four principal components. The eigenvectors corresponding to the four eigenvalues are listed in Table 3.

Then, the four principal components are described as $F_{i}=\mathbf{a}_{1 i} X_{1}^{*}+\mathbf{a}_{2 i} X_{2}^{*}+$ $\cdots+\mathbf{a}_{9 i} X_{9}^{*}$, for example, the first principal components is presented as follows:

$$
\begin{aligned}
F_{1}= & 0.5036 X_{1}^{*}-0.2454 X_{2}^{*}-0.1634 X_{3}^{*}-0.3101 X_{4}^{*}+0.0702 X_{5}^{*} \\
& -0.1665 X_{6}^{*}-0.3872 X_{7}^{*}-0.4377 X_{8}^{*}+0.4349 X_{9}^{*}
\end{aligned}
$$

Then the score matrix $F$ and the total points of the 20 amino acids are obtained and listed in Table 4.

Table 2. The eigenvalues, contribution rates and the accumulative contribution rates.

\begin{tabular}{cccc}
\hline Number & $\lambda$ & CR & ACR \\
\hline 1 & 3.2237 & 0.3582 & 0.3582 \\
2 & 1.9132 & 0.2126 & 0.5708 \\
3 & 1.4048 & 0.1561 & 0.7269 \\
4 & 1.1876 & 0.1320 & 0.8588 \\
5 & 0.4959 & 0.0551 & 0.9139 \\
6 & 0.4467 & 0.0496 & 0.9635 \\
7 & 0.1992 & 0.0221 & 0.9857 \\
8 & 0.1218 & 0.0135 & 0.9992 \\
9 & 0.0071 & 0.0008 & 1.0000 \\
\hline
\end{tabular}

Table 3. The elements of the eigenvectors.

\begin{tabular}{rrrr}
\hline \multicolumn{1}{c}{$a_{1}$} & \multicolumn{1}{c}{$a_{2}$} & \multicolumn{1}{c}{$a_{3}$} & \multicolumn{1}{c}{$a_{4}$} \\
\hline 0.5036 & 0.1436 & 0.0571 & 0.2158 \\
-0.2454 & -0.1875 & 0.2304 & 0.6547 \\
-0.1634 & 0.1820 & 0.6298 & 0.2288 \\
-0.3101 & -0.1883 & -0.3964 & 0.5071 \\
0.0702 & 0.6464 & -0.0786 & 0.0532 \\
-0.1665 & 0.4465 & -0.5280 & 0.1877 \\
-0.3872 & 0.3931 & 0.1003 & -0.0532 \\
-0.4377 & 0.1844 & 0.2544 & -0.2273 \\
0.4349 & 0.2643 & 0.1738 & 0.3495 \\
\hline
\end{tabular}


Table 4. The elements of the score matrix and the total points of the amino acids.

\begin{tabular}{crrrrr}
\hline Symbol & S1 & \multicolumn{1}{c}{$\mathrm{S} 2$} & $\mathrm{~S} 3$ & \multicolumn{1}{c}{$\mathrm{S} 4$} & \multicolumn{1}{c}{$\mathrm{TP}$} \\
\hline $\mathrm{A}$ & -2.6914 & -0.2241 & 0.7502 & -0.2868 & -0.9324 \\
$\mathrm{C}$ & -0.2117 & -2.0889 & -1.4026 & 1.0634 & -0.5985 \\
$\mathrm{D}$ & 0.2342 & -1.9671 & -1.0269 & -1.3364 & -0.6709 \\
$\mathrm{E}$ & 0.2161 & -1.1982 & 0.2191 & -0.6557 & -0.2296 \\
$\mathrm{~F}$ & 1.6369 & -0.7903 & -0.3388 & 0.4879 & 0.4298 \\
$\mathrm{G}$ & -2.8770 & -0.2695 & 0.3369 & -1.0823 & -1.1780 \\
$\mathrm{H}$ & 2.1245 & -0.0444 & -1.2399 & -0.8369 & 0.4476 \\
$\mathrm{I}$ & -0.5598 & -0.2612 & 1.2524 & 1.5462 & 0.1435 \\
$\mathrm{~K}$ & 1.1324 & 2.4599 & -0.3867 & -0.6164 & 0.7868 \\
$\mathrm{~L}$ & -1.9755 & 0.8575 & 1.9353 & 0.7785 & -0.1205 \\
$\mathrm{M}$ & 1.4097 & -0.7517 & 0.6791 & 0.9274 & 0.5735 \\
$\mathrm{~N}$ & 0.9764 & -0.5803 & -0.1437 & -1.7290 & -0.0242 \\
$\mathrm{P}$ & -2.4070 & 0.7955 & -3.5031 & 1.9525 & -0.9822 \\
$\mathrm{Q}$ & 1.1724 & -0.2717 & 0.2473 & -0.8789 & 0.2848 \\
$\mathrm{R}$ & 0.9832 & 4.1089 & -0.4523 & -0.2891 & 1.1169 \\
$\mathrm{~S}$ & -2.0600 & 0.6987 & 0.2926 & -1.2430 & -0.7077 \\
$\mathrm{~T}$ & -0.9005 & -0.2815 & 0.0018 & -0.5338 & -0.4525 \\
$\mathrm{~V}$ & -1.5026 & -0.1298 & 1.1941 & 0.8726 & -0.2643 \\
$\mathrm{~W}$ & 3.1302 & -0.0067 & 0.9381 & 1.5664 & 1.4729 \\
$\mathrm{Y}$ & 2.1692 & -0.0551 & 0.6471 & 0.2936 & 0.9050 \\
\hline
\end{tabular}

\subsection{Graphical representation of protein sequences}

Let $\Omega=\{A, C, D, E, F, G, H, I, K, L, M, N, P, Q, R, S, T, V, W, Y\}, \quad \Psi=$ $p_{1} p_{2} p_{3} \ldots p_{N}\left(p_{i} \in \Omega, \forall i \in\{1,2, \ldots, N\}\right)$ represent a protein sequence with $N$ amino acids, then, we can obtain a time series $S_{\Psi}$ as we replace each letter $p_{i}$ in the $\Psi$ with its corresponding value of TP. Since the length of the time series is not the same, we will use fast Fourier transform convert the time series into sequences of complex numbers with the same length.

For the first 10 amino acids of the ND5 protein sequences of human, gorilla and opossum, we can get three time sequences and their corresponding spectrums,

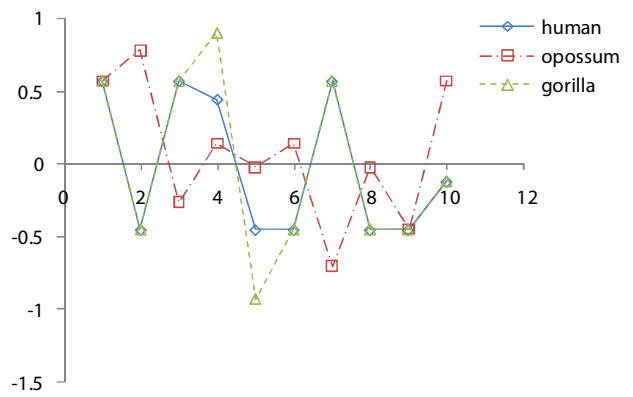

(a)

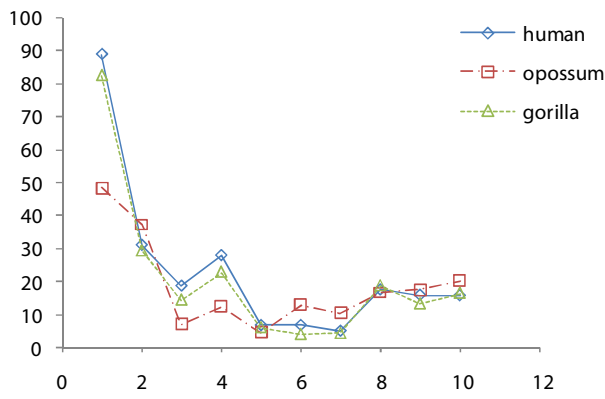

(b)

Fig. 1. (a) The time series for the first 10 amino acids of the ND5 proteins of human, opossum and gorilla. (b) The corresponding part of their FFT spectrums. 
which are shown in Fig. 1

$\mathrm{Hu}=$ MTMHTTMTTL; $\quad$ Gor = MTMYATMTTL; Opo = MKVINISNTM.

$\mathrm{S}_{\mathrm{Hu}}=\{0.5735,-0.4525,0.5735,0.4476$,

$$
-0.4525,-0.4525,0.5735,-0.4525,-0.4525,-0.1205\} \text {; }
$$

$\mathrm{S}_{\mathrm{Gor}}=\{0.5735,-0.4525,0.5735,0.9050$,

$$
-0.9324,-0.4525,0.5735,-0.4525,-0.4525,-0.1205\} \text {; }
$$

$\mathrm{S}_{\mathrm{Opo}}=\{0.5735,0.7868,-0.2643,0.1435$,

$$
-0.0242,0.1435,-0.7077,-0.0242,-0.4525,0.5735\} \text {. }
$$
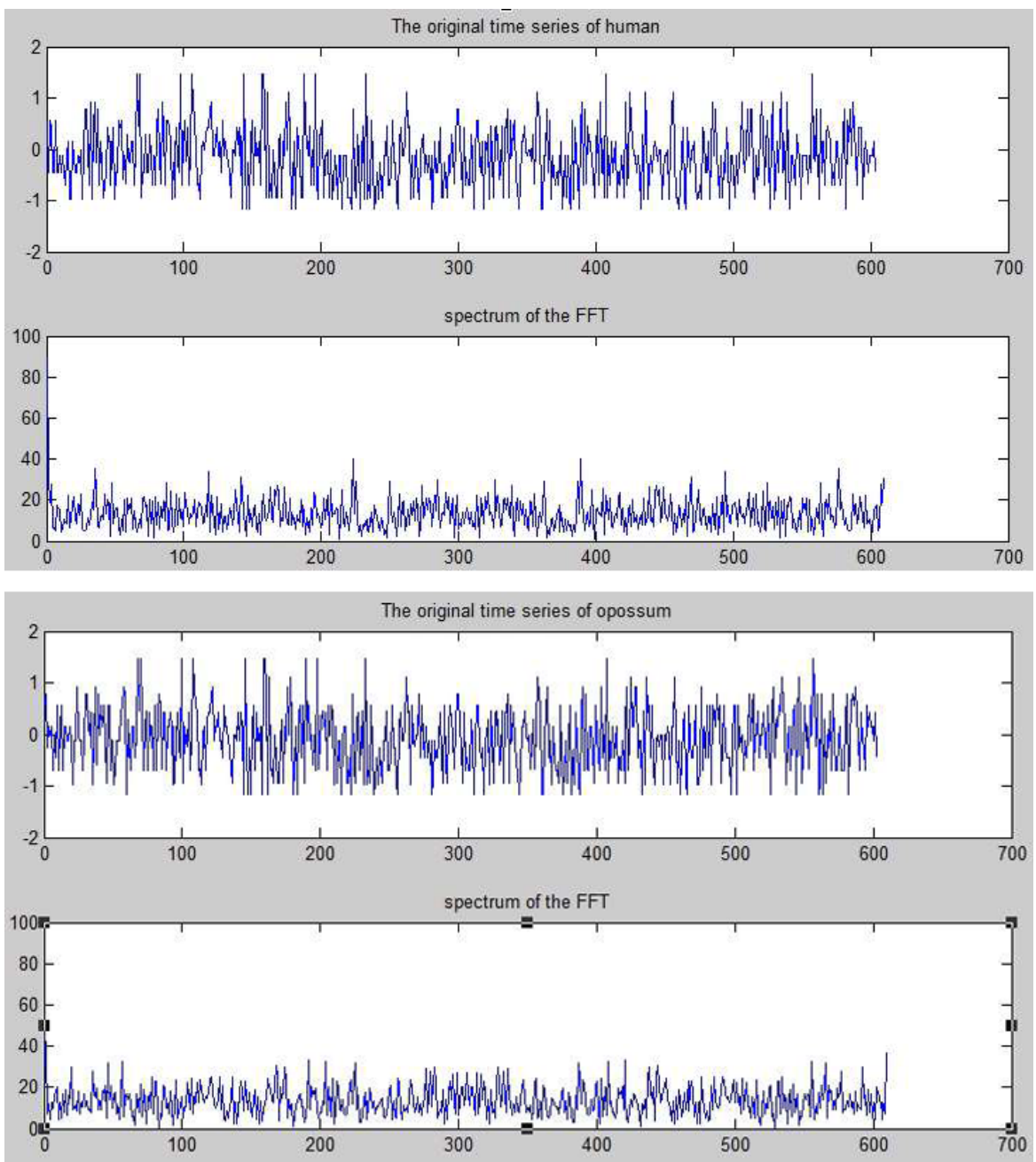

Fig. 2. The time series of human and opossum and their spectrums after the FFT method. 
From Fig. 1(a), we can visualize the similarity/dissimilarity between the three time sequences, and the result shows that protein sequences of human and gorilla are similar and protein sequences of human and opossum are dissimilar when we observe the curves carefully. After using the FFT, it shows the similarity between human and gorilla and the dissimilarity between human and opossum vividly and obviously, which is described in Fig. 1(b). It is obvious that the result in Fig. 1(b) is more visual and clear than that in Fig. 1(a).

The full sequences and their corresponding FFT spectrum of the ND5 protein sequences of human and opossum are plotted in Fig. 2. Apparently from Fig. 2, the similarity/dissimilarity between the two protein sequences are not distinguished easily. In the next section, we utilize the Euclidean distance between the curves and the cumulative difference between the spectrums of the corresponding protein sequences to compute the similarity/dissimilarity between the sequences respectively.

\section{Results and Discussion}

\subsection{Method for similarity analysis of protein sequences based on the PCA-FFT method}

To quantify comparisons of the protein sequences, in this section, we propose a new method for similarity analysis based on the distance between the time series and the complex sequences after the utilizing the FFT on them.

Firstly, for any two protein sequences $\Psi_{1}$ and $\Psi_{2}$, let $\left\{x_{1}, x_{2}, \ldots, x_{n 1}\right\}$ and $\left\{y_{1}, y_{2}, \ldots, x_{n 2}\right\}$ be their original time series respectively, then, we can define the distance between $\Psi_{1}$ and $\Psi_{2}$ as follows:

$$
d\left(\Psi_{1}, \Psi_{2}\right)=\sqrt{\sum_{i=1}^{N}\left(x_{i}-y_{i}\right)^{2}},
$$

where $N=\max$ (length $\left(\Psi_{1}\right)$, length $\left(\Psi_{2}\right)$ )

Obviously, the distance between two protein sequences can be used as an index for comparison of these two protein sequences, and the shorter the distance between them, the more similar these two protein sequences will be.

Based on the distance between two protein sequences defined above, the basic information of the 16 ND5 proteins is presented in Table 5, and the similarity/dissimilarity matrix is listed in Table 6 .

Observe Table 6 , it is easy to find that there are some similar pairs such as Fin Whale-Blue Whale (with distance of 3.3013), Pigmy Chimpanzee-Common Chimpanzee (with distance of 3.4477), Human-Common Chimpanzee (with distance of 4.4890), Sheep-Goat (with distance of 4.5737), Human-Pigmy Chimpanzee (with distance of 4.7036), hare-rabbit (with distance of 4.9311). Specially, the Opossum and gallus seem to be two peculiar animals since the shortest distance between then and the remaining mammals is more than 15.00, which is consistent with the fact 
Table 5. The basic information of 16 ND5 protein sequences.

\begin{tabular}{clllc}
\hline No. & Name & Abbreviation & Access No. & Length \\
\hline 1 & Human & human & ADT80430.1 & 603 \\
2 & Gorilla & gorilla & NP_008222 & 603 \\
3 & Pigmy Chimpanzee & pi-chim & NP_008209 & 603 \\
4 & Common Chimpanzee & c-chim & NP_008196 & 603 \\
5 & Fin Whale & f-whale & NP_006899 & 606 \\
6 & Blue Whale & b-whale & NP_007066 & 606 \\
7 & Rat & rat & AP_006899 & 610 \\
8 & Mouse & mouse & NP_904338 & 607 \\
9 & Opossum & opossum & NP_007105 & 602 \\
10 & Sheep & sheep & ABW22903.1 & 606 \\
11 & Goat & goat & BAN59258.1 & 606 \\
12 & Lemur & lemur & CAD13431.1 & 603 \\
13 & Cattle & cattle & ADN11902.1 & 606 \\
14 & Hare & hare & CAD13291.1 & 603 \\
15 & Gallus & gallus & BAE16036.1 & 605 \\
16 & Rabbit & rabbit & NP_007559.1 & 603 \\
\hline
\end{tabular}

that Opossum is the most remote species from the remaining mammals and the Gallus is not a mammal.

If we use the complex sequences of the time series after applying FFT on them, let $\left\{c 1_{1}, c 1_{2}, \ldots, c 1_{N}\right\}$ and $\left\{c 2_{1}, c 2_{2}, \ldots, c 2_{N}\right\}$ be the two complex sequences of the protein sequences $\Psi_{1}$ and $\Psi_{2}$ then we can compute the distance between the proteins in the following way.

$$
d\left(\Psi_{1}, \Psi_{2}\right)=\sum_{i=1}^{N} a b s\left(c 1_{i}-c 2_{i}\right),
$$

where $c 1_{i}$ and $c 2_{i}$ are complex numbers and $a b s\left(c 1_{i}-c 2_{i}\right)$ computes the module of the difference between $c 1_{i}$ and $c 2_{i}$. We list the similarity/dissimilarity matrix for the 16 ND5 proteins based on the distance between the complex sequences of protein sequences in Table 7 .

Obviously, the same similar pairs are shown in Table 7, so the FFT will not change the inner structure of the original time series, and we can consider the protein sequences as the signal sequences for our research.

\subsection{The phylogenetic tree of the protein sequences based on the PCA-FFT method}

A phylogenetic tree or evolutionary tree is a branching diagram or "tree" showing the inferred evolutionary relationships among various biological species or other entities and their phylogeny based upon similarities and differences in their physical and/or genetic characteristics. It is a commonly used tool by researchers in some fields to help and analyze the clustering of different species. We construct a phylogenetic tree of the above 16 ND5 proteins based on our PCAFFT method. We use the time series-based similarity matrix and the complex 


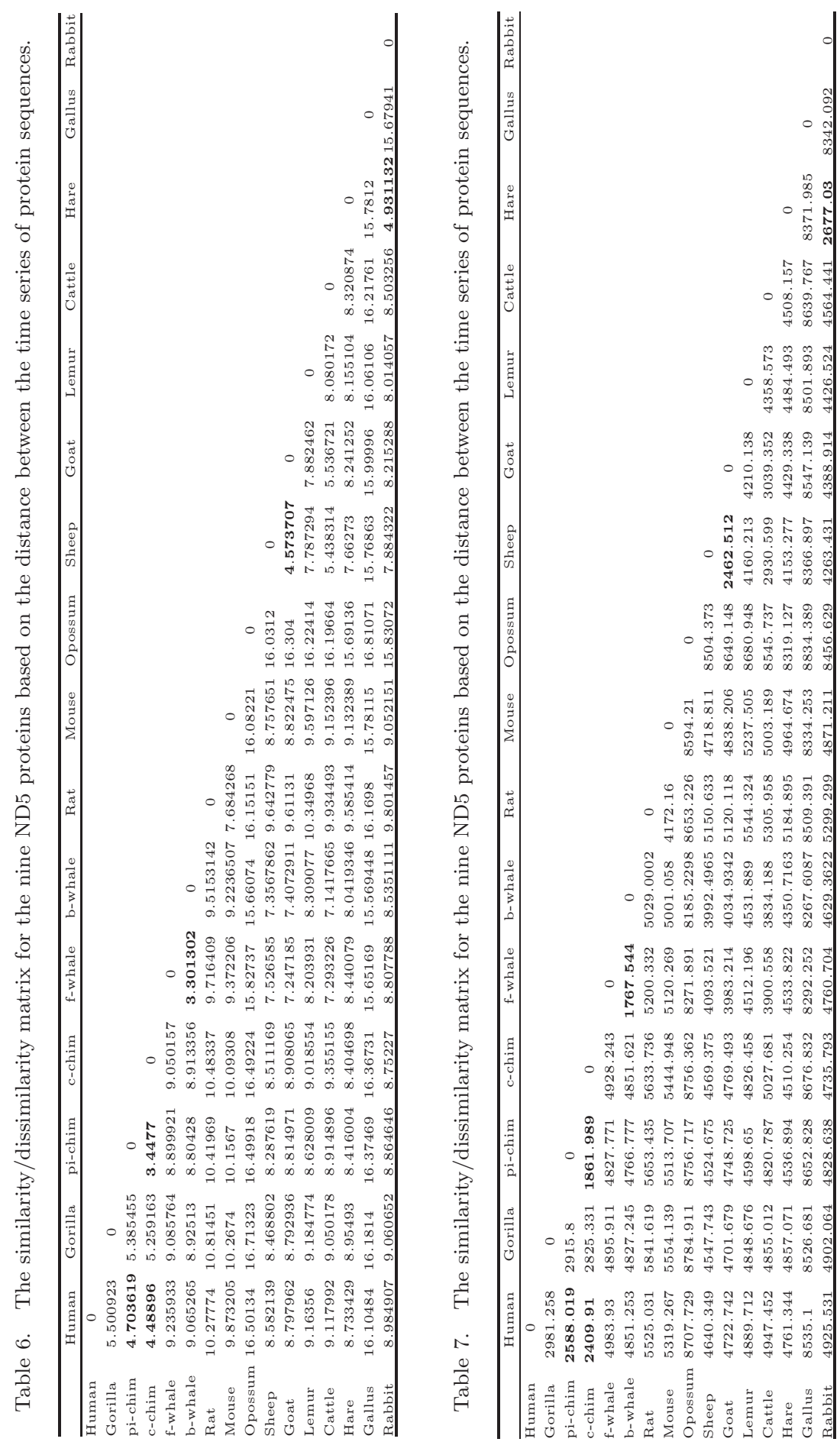




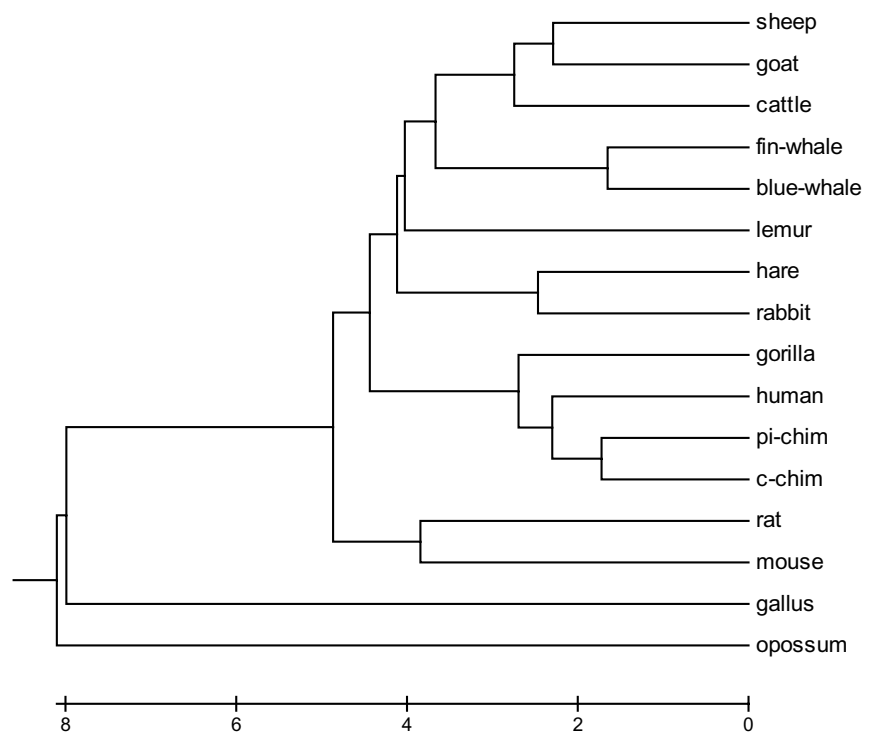

Fig. 3. The phylogenetic tree of the 16 species based on the PCA-FFT analysis of their ND5 protein sequences.

sequence-based similarity matrix as input data, respectively, and we got the same phylogenetic trees applying the UPGMA (Unweighted Pair Group Method with Arithmetic Mean) method, ${ }^{27}$ which is a simple agglomerative hierarchical clustering method that can be used to construct a phylogenetic tree. The phylogenetic tree based our method is presented in Fig. 3.

From Fig. 3, we can find that our method successfully clusters these 16 species based on the PCA-FFT analysis of their ND5 protein sequences. Specifically, the result shows that the gallus is peculiar, since the distance between it and the remaining animals is shortest. Obviously, the result is consistent with the fact that gallus is not a kind of mammal.

To validate the effectiveness of our method, we applied our method to another group of proteins that exist in a virus called spike protein ( $\mathrm{S}$ protein), which is a large type I transmembrane protein ranging from 1160 amino acids for avian infectious bronchitis virus (IBV) and up to 1400 amino acids for feline coronavirus $(\mathrm{FCoV})$. In addition, spike protein is highly glycosylated as it contains $21-35 \mathrm{~N}$ glycosylation sites. The basic information of these proteins is presented in Table 8 .

According to the above method, we construct the phylogenetic tree of these 29 spike proteins in Fig. 4.

The viruses in Table 8 are Coronavirus, they are always classified into four classes, where Group I includes the Canine coronavirus (CCoV), the Feline coronavirus (FCoV), the Human coronavirus 229E (HCoV-229E), the Porcine epidemic diarrhea virus (PEDV), the Transmissible gastroenteritis virus (TGEV) and so 


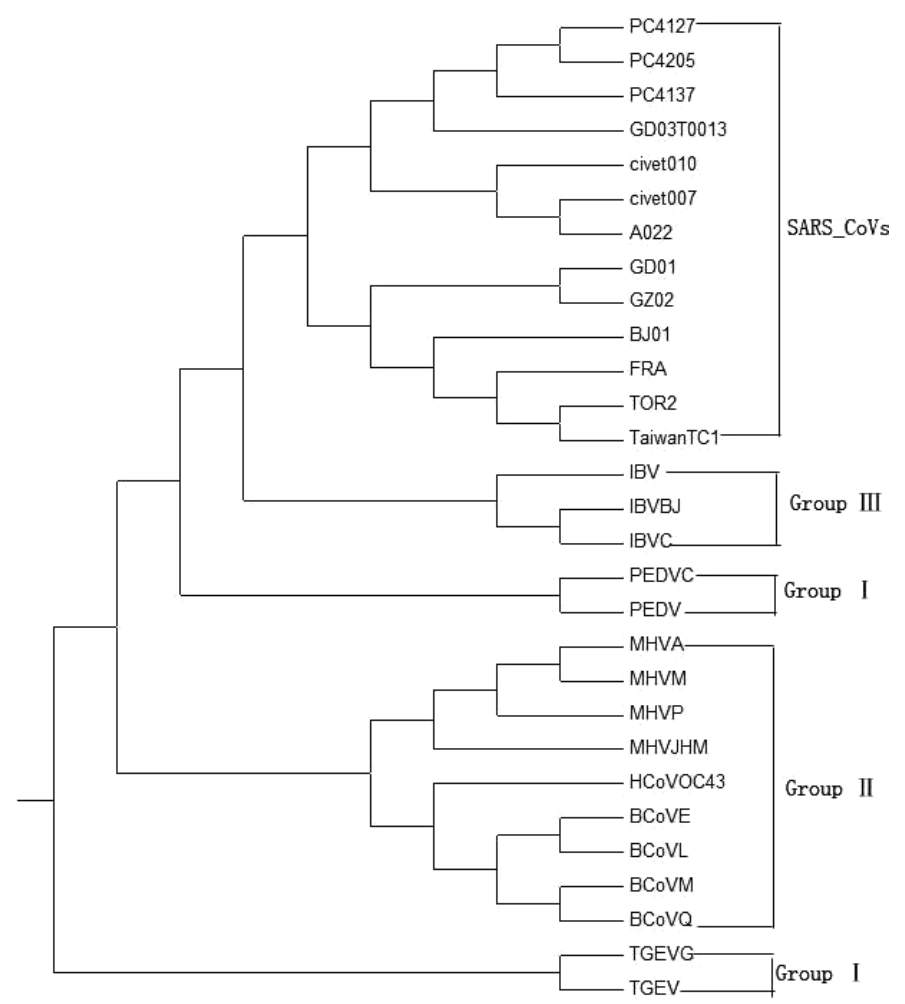

Fig. 4. The phylogenetic tree of these 29 spike proteins.

on. Group II includes the Bovine coronavirus (BCoV), Human coronavirus OC43 (HCoV-OC43), the Murine hepatitis virus (MHV), the Porcine hemagglutinating encephalomyelitis virus (HEV), the Rat coronavirus (RtCoV) and so on. Group III contains the Infectious bronchitis virus (IBV), the Turkey coronavirus (TCoV) and so on. The others are Severe Acute Respiratory Syndrome Coronaviruses (SARS$\mathrm{CoV}$ ). In Fig. 4, we can observe that the SARS-CoVs, Groups II and III can be separated correctly, however, Group I has been broken into two parts.

Even though the classification of Group I is broken into two parts, it is still a new method for helping to analyze the similarity/dissimilarity of protein sequences. Besides, comparing with other methods, our method analyzes similarity/dissimilarity of protein sequences based on nine properties, rather than two or three. For example, Hou et al. ${ }^{5}$ consider two properties of amino acids as the descriptor to reflect the relation among different proteins, Elmaatv et al. ${ }^{6}$ provide the method for correlation analysis based on three physicochemical properties. Then the PCA-FFT is utilized for reducing the dimension and transforming the protein sequences into number series to the correlation analysis. 
Table 8. The basic information of 29 spike proteins.

\begin{tabular}{rllc}
\hline No. & Access No. & Abbreviation & Length \\
\hline 1 & CAB91145 & TGEVG & 1447 \\
2 & NP_058424 & TGEV & 1447 \\
3 & AAK38656 & PEDVC & 1383 \\
4 & NP_598310 & PEDV & 1383 \\
5 & NP_937950 & HCoVOC43 & 1361 \\
6 & AAK83356 & BCoVE & 1363 \\
7 & AAL57308 & BCoVL & 1363 \\
8 & AAA66399 & BCoVM & 1363 \\
9 & AAL40400 & BCoVQ & 1363 \\
10 & AAB86819 & MHVA & 1324 \\
11 & YP_209233 & MHVJHM & 1376 \\
12 & AAF69334 & MHVP & 1321 \\
13 & AAF69344 & MHVM & 1324 \\
14 & AAP92675 & IBVBJ & 1169 \\
15 & AAS00080 & IBVC & 1169 \\
16 & NP_040831 & IBV & 1162 \\
17 & AAS10463 & GD03T0013 & 1255 \\
18 & AAU93318 & PC4127 & 1255 \\
19 & AAV49720 & PC4137 & 1255 \\
20 & AAU93319 & PC4205 & 1255 \\
21 & AAU04646 & civet007 & 1255 \\
22 & AAU04649 & civet010 & 1255 \\
23 & AAV91631 & A022 & 1255 \\
24 & AAP51227 & GD01 & 1255 \\
25 & AAS00003 & GZ02 & 1255 \\
26 & AAP30030 & BJ01 & 1255 \\
27 & AAP50485 & FRA & 1255 \\
28 & AAP41037 & TOR2 & 1255 \\
29 & AAQ01597 & TaiwanTC1 & 1255 \\
\hline & & &
\end{tabular}

Moreover, to demonstrate the effectiveness of our method, the similarities of ND5 proteins of nine species are compared. Clustal W is one of most popular multiple sequence alignment methods which uses sequence weighting, position specific gap penalties and weight matrix choice to improve the sensitivity of progressive multiple sequence alignment. ${ }^{28}$ For comparing with our results, the scatter diagrams are plotted to obtain the results of other graphical representation correlation with Clustal W's results in Fig. 5. In these scatter diagrams, the linear lines are given. If the points are all around the trend line, this shows that the correlation is better. Observing the figure, our method presented higher correlation with Clustal $\mathrm{W}$ than the methods of Hou et al., ${ }^{5}$ Elmaatv et al., ${ }^{6}$ Wen et al. method, ${ }^{7}$ Zhang et al. method, ${ }^{8} \mathrm{Wu}$ et al. ${ }^{9}$ Yao et al., ${ }^{10}$ and He et al. ${ }^{14}$ So in this paper, a new approach is provided to analyze the similarities/dissimilarities of protein sequence, which is comparatively more efficient. 

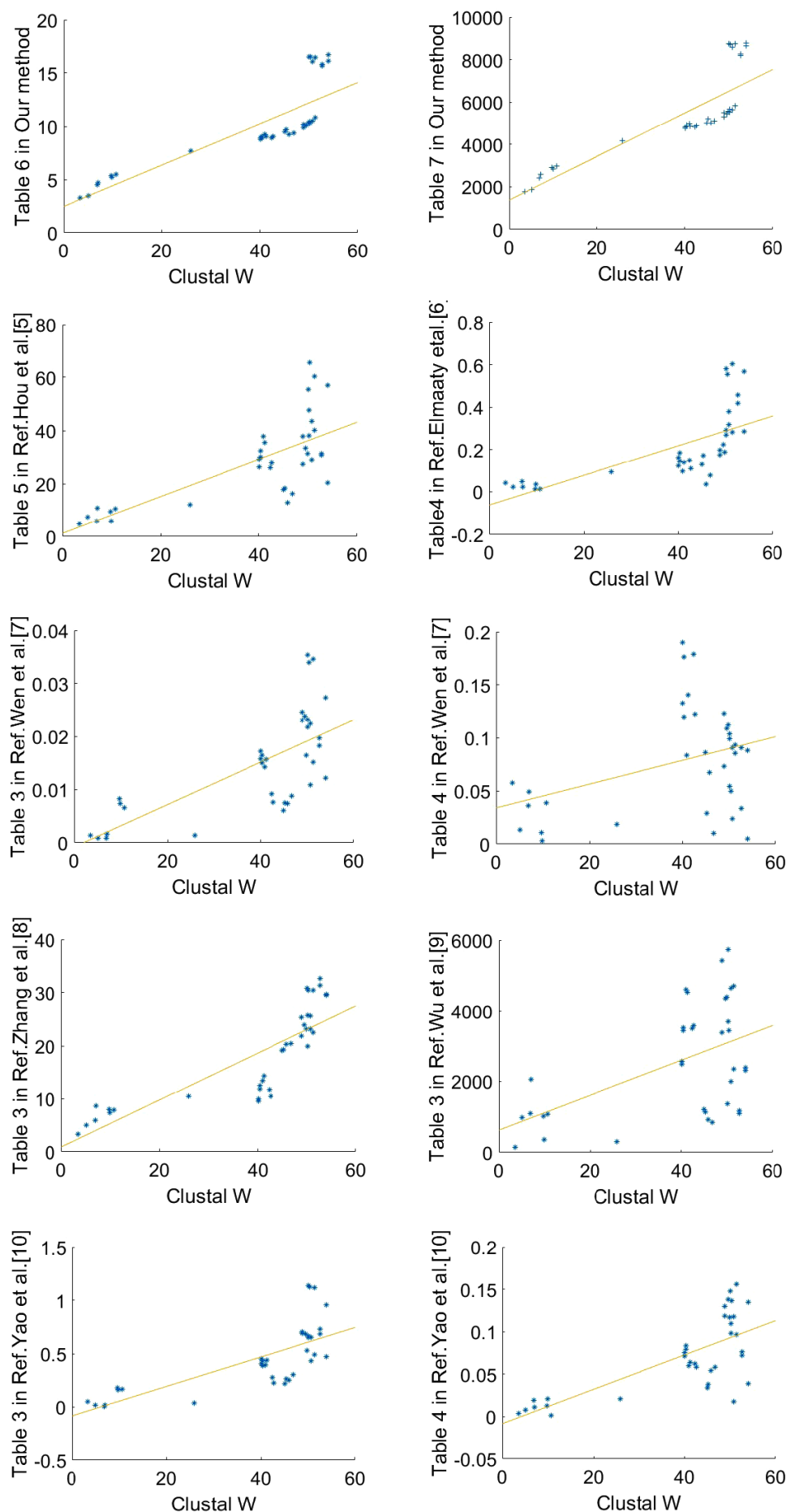

Fig. 5. Comparison of different methods by correlation analysis. 


\section{Conclusions}

In this paper, we apply the PCA to transform the protein sequences into time series. By this way, we can easily find the differences between protein sequences if we plot them in a same coordinate system. We consider the time series as signals and utilize the FFT to produce them, and then we get the information of their time-frequency features. We make use of the time series and their time-frequency features to analyze the similarity/dissimilarity of protein sequences, which not only contain more information of about the proteins, but also do not need to build the high dimensional matrixes which require complex computation. To observe the similarity more conveniently, we construct the phylogenetic tree of the similarity/dissimilarity matrix using the UPGMA method. Then two groups of sequences are chosen to validate our method, and we get the satisfying results. Finally, the scatter diagrams are constructed for comparing our method with some existing methods to validate the effectiveness of the method presented in this paper.

\section{Acknowledgments}

The authors thank the anonymous referees for suggestions that helped improve the paper substantially. And the project is partly sponsored by the Construct Program of the Key Discipline in Hunan province, the National Natural Science Foundation of China (No. 61640210, No. 61672447), the CERNET Next Generation Internet Technology Innovation Project (No. NGII20160305), and the Upgrading Project of Industry-University-Research of Xiangtan University (No. 11KZ|KZO3051).

\section{References}

1. Koonin EV, Galperin MY, Sequence-Evolution-Function: Computational Approaches in Comparative Genomics, Kluwer Academic Boston, 2003.

2. Wang L, Peng H, Zheng J et al., ADLD: A novel graphical representation of protein sequences and its application, Comput Math Methods Med 2014(2014):178-193, 2014.

3. Wang L, Peng H, Zheng J et al., Similarities/dissimilarities analysis of protein sequences based on recurrence quantification analysis, Cur Bioinform 10:112-119 2015.

4. Gates MA, Simpler DNA sequence representations, Nature 316(6025):219 (1985).

5. Hou W, Pan Q, He M et al., A new graphical representation of protein sequences and its applications, Phys A Stat Mech Appl 444:996-1002 (2016)

6. Maaty MI, Aboelkhier MM, Elwahaab MA et al., 3D graphical representation of protein sequences and their statistical characterization, Phys A Stat Mech Appl 389(21):4668-4676 (2010).

7. Wen J, Zhang Y, A 2D graphical representation of protein sequence and its numerical characterization, Chem Phys Lett 476(4):281-286, 2009.

8. Zhang Y, Ruan J, He P et al., Analyzes of the similarities of protein sequences based on the pseudo amino acid composition, Chem Phys Lett 590(6):239-244, 2013.

9. Wu Z, Xiao X, Chou K et al., 2D-MH: A web-server for generating graphic representation of protein sequences based on the physicochemical properties of their constituent amino acids, J Theor Biol 267(1):29-34, 2010. 
10. Yao Y, Dai Q, Li C et al., Analysis of similarity/dissimilarity of protein sequences, Proteins 73(4):864-871, 2008.

11. $\mathrm{Mu} \mathrm{Z}, \mathrm{Wu}$ J, Zhang $\mathrm{Y}$ et al., A novel method for similarity/dissimilarity analysis of protein sequences, Phys A Stat Mech Appl 392(24):6361-6366, 2013.

12. Yao Y, Kong F, Dai Q et al., A sequence-segmented method applied to the similarity analysis of long protein sequence, MATCH Commun Math Comput Chem 70:431-450, 2013.

13. Liu L, Li D, Bai F et al., A relative Lempel-Ziv complexity: Application to comparing biological sequences, Chem Phys Lett 530:107-112, 2012.

14. Liu Y, Li D, Lu K et al., P-H Curve, a graphical representation of protein sequences for similarities analysis, MATCH Commun Math Comput Chem 70:451-466, 2013.

15. Ellakkani A, Elsherif S, Similarity analysis of protein sequences based on 2D and 3D amino acid adjacency matrices, Chem Phys Let 590:192-195, 2013.

16. Ma T, Liu Y, Dai Q et al., A graphical representation of protein based on a novel iterated function system, Phys A Stat Mech Appl 403:21-28, 2014.

17. Yao Y, Yan S, Han J et al., A novel descriptor of protein sequences and its application, $J$ Theor Biol 347:109-117, 2014.

18. Balsera MA, Wriggers W, Oono Y et al., Principal component analysis and long time protein dynamics, J Phys Chem 100:2567-2572, 1996.

19. Hess B, Similarities between principal components of protein dynamics and random diffusion, Phys Rev E Stat Phys Plasmas Fluids Relat Interdiscip Topics $62(6 P+B)$ : 8438-8448, 2000.

20. Tournier A, Smith JC, Principal components of the protein dynamical transition, Phys Rev Lett 91:208106, 2003.

21. Ritchie DW, Venkatraman V, Ultra-fast FFT protein docking on graphics processors, Bioinformatics 26(19):2398-2405, 2010.

22. Huang P, Love JJ, Mayo SL et al., Adaptation of a fast Fourier transform-based docking algorithm for protein design, J Comput Chem 26(12):1222-1232, 2005.

23. https://www.mcb.ucdavis.edu/courses/bis102/AAProp.html.

24. Boye JI, Zare F, Pletch A et al., Pulse proteins: Processing, characterization, functional properties and applications in food and feed, Food Res Int 43(2):414-431, 2010.

25. https://en.wikipedia.org/wiki/Amino_acid\#cite_note-136.

26. Pearson K, On lines and planes of closest fit to systems of points in space, Philos. Mag 2:559-572, 1901.

27. Sokal RR, Michener CD, A statistical method for evaluating systematic relationships, Univ Kansas Sci Bull 38(2):1409-1438, 1958.

28. Thompson JD, Higgins DG, Gibson TJ et al., CLUSTAL W: Improving the sensitivity of progressive multiple sequence alignment through sequence weighting, positionspecific gap penalties and weight matrix choice, Nucleic Acids Res 22(22):4673-4680, 1994. 